\title{
HMGA2 gene silencing suppression SW480 colon cancer cell invasion and expression of Survivin
}

\author{
Ping WANG ${ }^{1}$ Shou xun WANG ${ }^{2}$ Jun hong DONG ${ }^{3^{*}}$ \\ Biochemistry office, Weifang Medical College,Weifang 261042, China

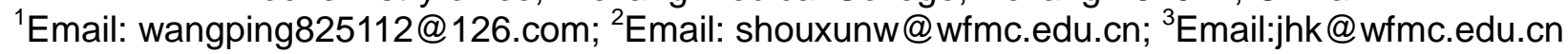 \\ ${ }^{*}$ corresponding author
}

Keywords: RNA interference; Survivin; HMGA2; p53

\begin{abstract}
Objective To investigate the effects of high mobility group protein A2 (HMGA2) gene interference SW480 cell invasion, and to explore its interference with the expression of Survivin gene and its mechanism.Method: To construct the recombinant HMGA2 small hairpin interfering RNA expression vector pGenesil-1.1- HMGA2, SW480 colon cancer cells were transfected, cell growth were detected by MTT, the changing of cell invasion ability were detected by Transwell chamber invasion assay; The mRNA and protein expression of Survivin, HMGA2 and P53 were detected by PCR and Western-blot. Construction of RNA interference vectors which target P53 gene ,transfection SW480 cells, detect the mRNA and protein expression of Survivin Result: After the HMGA2 gene interference, SW480 cell proliferation was significantly inhibited, inhibition rate was $34.44 \%$, cell invasion was significantly inhibited, The mRNA and protein expression levels of Survivin and P53 genes significantly reduced. After P53 gene is disturbed, mRNA and protein expression levels of Survivin gene decline. Conclusion: Targeting and silencing HMGA2 gene have significantly inhibition for SW480 cells; while down Survivin gene expression, which may be related to down-regulation of gene expression in P53.
\end{abstract}

\section{Introduction}

HMGA2 is a structure transcription factor, has not transcriptional activation, but can adjust the transcription and activation for a large number of gene, particularly those genes which associated with cell proliferation and apoptosis, plays important role in tumorigenesis, invasion and metastasis, but the exact mechanism is unclear [1]Survivin is apoptosis Inhibition gene, recently found that high expression of Survivin is also closely associated with tumor invasion and metastasis, suggesting tumor recurrence, lymph node invasion and distant metastasis increased risk [2,3]. It has been suggested both HMGA2 and Survivin gene expression may have some relevance to jointly regulate tumor progression [4]. This HMGA2 gene targeting shRNA interference vector was designed and constructed, By HMGA2 gene inhibition of SW480 cell proliferation and invasion, and to explore the impact of expression of Survivin in SW480 cells by interference HMGA2, which may be associated with reduced P53 down-regulation.

\section{Materials and reagents}

Human colon cancer cell line SW480 (Chinese Academy of Sciences Shanghai Cell Bank); pGenesil-1.1 plasmid (Wuhan Jing game company); plasmid extraction kit (QIAGEN Company USA); FuGENE HD transfection reagent and TRAP-ELISA kit (Roche Inc.); monoclonal Anti-Survivin 1 antibody (CST company), monoclonal anti-HMGA2, and anti-P53 antibody (Santa Cruz company). Matrigel matrix synthetic rubber (US BD Biosciences), and interfere sequence and the primer sequences (Beijing Genomics Kuni company).

Construction siRNA expression vector. According to the GenBank HMGA2 (NM_003483) and P53 (NM_00546.4) cDNA sequence, Three groups were designed to interfere with the sequence, 
respectively. Preliminary experimental screening of highly efficient and specific sequence of interference, HMGA2 interference targeting sequence 5'-CGCCAACGTTCGATTTCAT-3 ', P53 interference targeting sequence 5'-GACTCCAGTGGTAATCTAC -3' And to design a non-specific sequence 5-AAGACTTCATAAGGCGCATGC-3 'as a negative control. Respectively,the HMGA2 and P53 annealed fragments were connected with the linearized vector pGenesil-1.1, namely pGenesil-1.1- HMGA2 and pGenesil-1.1- P53, ; negative control product that is connected with vector, namely pGenesil-1.1-HK.

Recombinant plasmid transfected SW480 colon cancer cells. SW480 cells were cultured at $37^{\circ} \mathrm{C}$ and without $\mathrm{CO} 2$ in L15 medium (Beijing Mai Chen Company), supplemented with $10 \%$ heat inactivated fetal bovine serum (Hyclone Thermo Scientific, Scoresby, Victoria, Australia, FBS), $100 \mathrm{U} / \mathrm{ml}$ penicillin and $100 \mu \mathrm{g} / \mathrm{ml}$ streptomycin.24h before transfection of SW480 cells in logarithmic growth phase $5 \times 10^{5}$ inoculation in 6-well plates, when the cells were grown to $80 \%$ confluence according to the instructions transfection. The experimental was divided into a control group (without any treatment), the negative control group (transfected pGenesil-1.1-HK), RNAi group (transfected pGenesil-1.1- HMGA2) and RNAi group (transfected pGenesil-1.1-P53).

Changes in cell proliferation HMGA2 gene detected interference MTT. The SW480 cells were transfected with pGenesil-1.1- HMGA2 or negative control plasmid, Each group of three wells, respectively 24, 48 and 72h after transfection, adding MTT solution (5g / L) 10 $\mu$, and incubated for $4 \mathrm{~h}$ in Incubator, then each well was added $100 \mu \mathrm{DMSO}$, shaken to fully dissolve crystals, each well was measured with microplate at $570 \mathrm{~nm}$ wavelength, the experiment was presented from at least three independent experiments, Calculation of cell proliferation inhibition rate $(\%)=(1-\mathrm{A}$ values in the experimental group / control group A value) $\times 100 \%$.

HMGA2 gene changes in cell invasion ability after disturbance. Matrigel glue thrawed overnight at $4{ }^{\circ} \mathrm{C}$ and diluted Matrigel glue, diluted to $1: 8$ with cold serum-free 1640 . Then $100 \mu \mathrm{l}$ Matrigel glue add to the transwell chamber, chamber into a 24-well plate, $37{ }^{\circ} \mathrm{C}$ incubated for $4 \mathrm{~h}$ to become gel, logarithmic phase cells in each group, with a serum-free medium was adjusted to $1 \times$ $106 / \mathrm{ml}$ of cell suspension, $0.2 \mathrm{ml}$ cell suspension was added to take the upper chamber, the lower chamber was added $0.6 \mathrm{ml} 10 \%$ fetal bovine serum medium, each group has three wells. The chamber were removed after $24 \mathrm{~h}$, Matrigel gel and without pass through the cell were wiped with a cotton swab, paraformaldehyde, Giemsa staining, magnification 400 pictures, count cells number, each experiment data are presented from at least three independent experiments.

Western-blot detection of protein expression on RNA interference. SW480 cells were transfected with siRNA with FuGENE HD,48h after transfection, cells were harvested in RIPA buffer.Total protein was quantitated using the BCA method. $50 \mu \mathrm{g}$ protein per well samples were taken on the SDS-PAGE electrophoresis, and transferred to PVDF membrane, and blocked with 5\% nonfat dry milk in TBST for $1 \mathrm{~h}$, added primary antibody (rabbit anti- Survivin monoclonal antibody diluted 1: 1000; mouse anti- P53 monoclonal antibody and anti-HMGA2 1: 500; mouse anti- $\beta$-actin monoclonal antibody at 1: 1000) and incubated overnight at $4{ }^{\circ} \mathrm{C}$, the next day were sed HRP-conjugated secondary IgG (1: 20,000 dilution) and incubated at room temperature for $1 \mathrm{~h}$, Chemiluminescence detection was performed using ECL, the protein concentration were analyzed.

Quantitative real-time PCR detection of RNA interference of mRNA.Total RNA from SW480 cells were extracted using a standard TRIzol RNA isolation method after 48 hours. The reverse transcription to cDNA and PCR experiments were performed with the Revertra Ace qPCR RT Kit, according to the manufacturer's protocols. The quantitative Real-time PCR were conducted in the LightCycler apparatus using the FastStart Universal SYBR Green Master. The expression value was normalized to $\Delta \Delta \mathrm{Ct}$ method. The sequences of the primer pairs are designed using Primer 5.0 software and provided in Table1 
Table 1 The primers used in this study

\begin{tabular}{|l|l|c|}
\hline \multicolumn{1}{|c|}{ name } & \multicolumn{1}{|c|}{ Sequences } & Amplicon Sizes(bp) \\
\hline Survivin & $\begin{array}{l}\text { 5' -GTCTGGCGTAAGATGATGGATTTG-3' } \\
\text { 5' -TCTCAGGAACAGCCGAGATGAC-3' }\end{array}$ & 113 \\
\hline P53 & $\begin{array}{l}\text { 5' -GCGTGTGGAGTATTTGGATGAC-3' } \\
\text { 5'-AGTGTGATGATGGTGAGGATGG-3' }\end{array}$ & 168 \\
\hline HMGA2 & $\begin{array}{l}\text { 5'-CCCAAAGGCAGCAAAACAA-3' } \\
\text { 5'-GCCTCTTGGCCGTTTTCTC-3' }\end{array}$ & 81 \\
\hline GAPDH & $\begin{array}{l}\text { 5' -GCACCGTCAAGGCTGAGAAC-3' } \\
\text { 5' -TGGTGAAGACGCCAGTGGA-3' }\end{array}$ & 138 \\
\hline
\end{tabular}

Statistical Analysis. Using SPSS11.0 software,Groups were compared by t test,The difference of $\mathrm{P}<0.05$ had statistical significance.

\section{Results}

Detect changes after Survivin gene interfere with cell proliferation with MTT. With the incubation time, the proliferation of SW480 cells was significantly slowed with Survivin gene interference, $24 \mathrm{~h}$ after the proliferation rate was significantly higher than the control group and negative control group $(\mathrm{P}<0.05)$, at $48 \mathrm{~h}$ inhibition rate reached $48.15 \%$ (Fig 1).

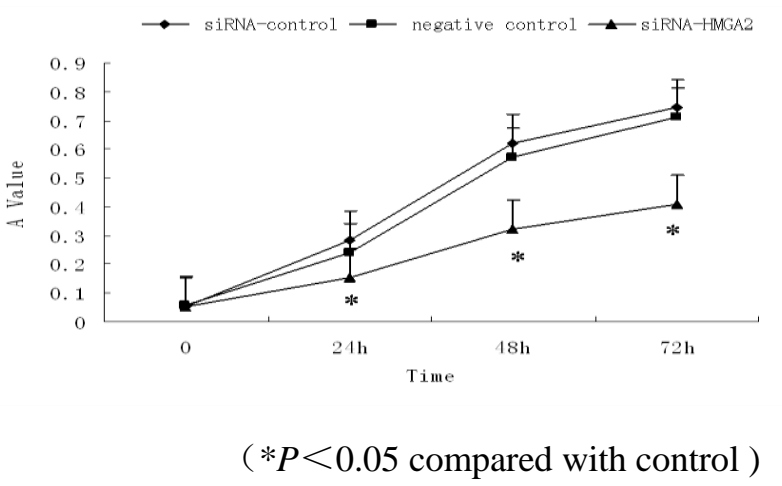

Figure. 1 Effects of transfected with siRNA- HMGA2 on proliferation of SW480 cells

HMGA2 RNA interference effects on cell invasion ability. HMGA2 gene interference effect on invasive ability of SW480 cells by transwell chamber invasion assay, the results shown in Figure 26.The control group cells through Matrigel gel cells (122.34 \pm 8.36$)$ / vision, negative control cells through Matrigel gel cells $(114.45 \pm 12.51)$ / vision, and siRNA -HMGA2 group through Matrigel gel cells $(64.47 \pm 7.51)$ / vision in $24 \mathrm{~h}$.With the blank control group, HMGA2 interference group of SW480 cells in vitro through the number of Matrigel glue is decreased obviously (P <0.05) (Fig 2).

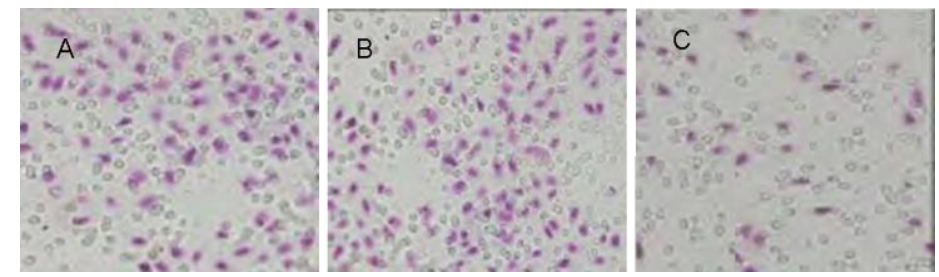
A. siRNA-control ;
B.negative -control ;
C.SiRNA-HMGA2

Figure. 2 Cell cycle distribution of SW480 cells transfected with siRNA- HMGA2 analyzed by flow cytometry

HMGA2 RNA interference affects on p53 and survivin gene expression. HMGA2 protein has 
significant interference by pGenesi-1.1- HMGA2 in SW480; Survivin and p53 gene expression was significantly decreased when the HMGA2 gene interference (Fig 3A); mRNA of Survivin and p53 were significantly lower than the control group and negative control group $(\mathrm{P}<0.01)$ (Fig $3 \mathrm{~B})$.

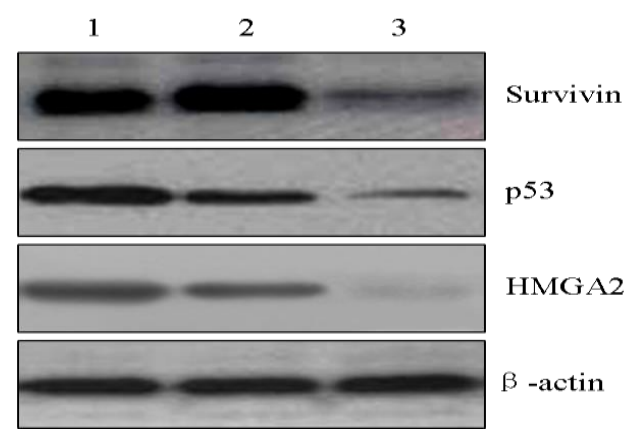

A
2

4

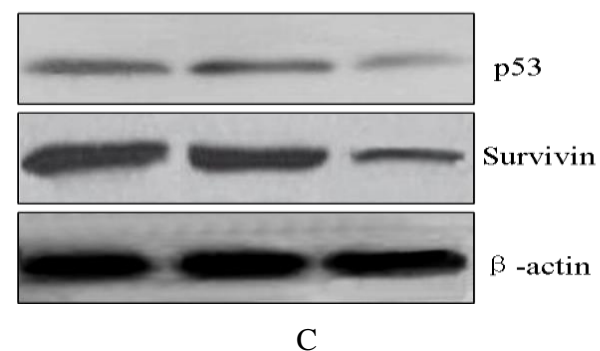

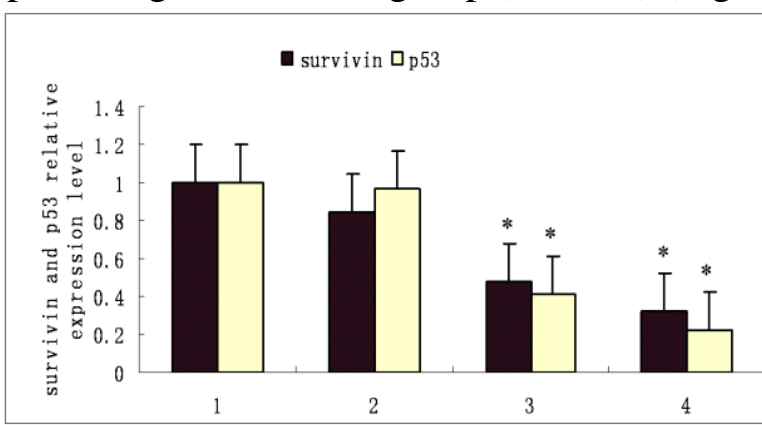

B

A. Western blot result of transfection with pGenesi-1.1- HMGA2; B. mRNA relative expression leves; C. Western blot result of transfection with pGenesi-1.1- p53; 1. siRNA-control; 2. negative -control; 3. siRNA-HMGA2; 4. siRNA-p 53; $* P<0.01$ compared with control

Figure. 3 Effects of shRNA on expressions of surviving and p53 in SW480 cells

P53 RNA interference affect on expression of surviving. After SW480 cells were transfected with pGenesil-1.1-p53, p53 protein expression was significantly reduced, while the expression of Survivin protein was also decreased (Fig 3C); mRNA expression of Survivin is also reduced (P <0.01) (Fig 3B ).

\section{Discussion}

HMGA2 is HMGA (high mobility group protein A), one of the family members, It showed high expression of all embryonic tissues, almost no expression in normal adult tissues, but in some malignant cells also reproduce the high expression of the embryonic period, It plays an important role in a variety of tumors occurrence, invasion and metastasis [1].Miyazawa et al [5] found that the stronger the invasive tumor, HMGA2 expression levels higher, accompanied by the loss of epithelial phenotype markers cadherin. Recent research suggest that it may be through the regulation of a variety of tumor formation and metastasis-related gene expression, but its exact mechanism rarely reported.In this work, the SW480 cells were transfected with HMGA2 RNA interference plasmid, the results shown to effectively inhibit the proliferation of SW480 cells, reducing the invasive ability of SW480 cells.

Members of the family of HMGA1 and HMGA2 were HMGA, their structure and function are similar. P53 protein is a transcription factor that regulates the expression of apoptotic genes able to induce apoptosis. Pierantoni studies have found, HMGA1 can combine with P53 in the body, and inhibite of BCL-2 associated X protein and cell cycle-dependent kinase inhibitor 1A (CDKN1A) transcription activator which are mediated by P53. MDM2 transcription level was inhibited when the P53 activation, this block the apoptosis of tumor cells [6,7]; But whether HMGA2 can be combined with P53, no studies have reported. However, studies have found, HMGA2 in invasive ovarian cancer tissue was significantly correlated with the expression level of P53, the progress of the 
process involved in ovarian cancer [8], it suggesting that HMGA2 may affect P53 expression .

In the present study that mRNA and protein expression of Survivin gene are significantly reduced when HMGA2 gene interference. It suggest HMGA2 regulate gene expression of Survivin. To further understand the mechanism of gene expression of Survivin gene down-regulation caused by HMGA2 interference. Through the mRNA and protein level of detection, we found that the p53 gene expression after reducing decreased after P53 interference, Survivin gene expression synchronization.Hoffman WH et al [9] found that Survivin promoter has p53 binding sites, by directly combining the p53 binding sites on the promoter inhibition of the expression of Survivin. This study confirmed that p53 silence can inhibit the expression of Survivin, suggesting that p53 involved in regulation of Survivin expression in SW480 cells.In addition, mRNA and protein expression levels of p53 reduced simultaneously after interference HMGA2 gene. These results suggest that HMGA2 gene interference cause reduce expression of Survivin by lower p53 expression, the exact mechanism remains to be further studied.

\section{Acknowledgements}

This research is supported by Shandong Province Science and Technology Program Higher (J14LK57); Shandong Province Natural Science Foundation funded project (ZR2014JL050, ZR2010HM065);Weifang Medical Technology Innovation Fund (K1302027) and the domestic visiting scholar fund of Weifang Medical College.

\section{References}

[1]Wang X, Liu X and Li AY. Overexpression of HMGA2 promotes metastasis and impacts survival of colorectal cancers, Clin Cancer Res. Vol. 17(2011), p.2570-80.

[2]Chu XY, Chen LB and Wang JH. Overexpression of survivin is correlated with increased invasion and metastasis of colorectal cancer, J Surg Oncol. Vol. 105(2012), p.520-8.

[3] Marioni G, Bertolin A and Giacomelli L. Expression of the apoptosis inhibitor proteinSurvivin in primary laryngeal carcinoma and cervical lymph node metastasis, AnticancerRes. Vol. 26(2006), p.3813-7.

[4] $\mathrm{Wu} \mathrm{T,} \mathrm{Jia} \mathrm{J}$ and Xiong $\mathrm{X}$. Increased expression of Lin28B associates with poor prognosis in patients with oral squamous cell carcinoma, PLoS One. Vol. 8(2013), p.e83869

[5]Miyazawa J, Mitoroa and Kawashiris.Expression of mesenchyme-specific gene HMGA2 in squamous cell carcinomas of the oral cavity, CancerRes. Vol. 64(2004), p.2024-2029.

[6]Pierantoni G M, Rinaldo C and Esposito F. High Mobility Group A1 (HMGA1) proteins interact with p53 and inhibit its apoptotic activity, Cell Death Differ. Vol.13(2006), p.1554-1563.

[7]Esposito F, Tornincasa M and Federico A. High-mobility group A1 protein inhibits p53-mediated intrinsic apoptosis by interacting with Bcl-2 at mitochondria, Cell Death Dis. Vol.25(2013), p.299-307.

[8] Mahajan A1, Liu Z and Gellert L. HMGA2: a biomarker significantly overexpressed in high-grade ovarian serous carcinoma, Mod Pathol. Vol. 23(2010), p.673-81.

[9] Hoffman WH, Biade S and Zilfou JT. Transcriptional repression of the anti-apoptotic survivin gene by wild type p53, J Biol Chem. Vol.277(2002), p.3247-57. 